\title{
Detection of COL1A1-PDGFB fusion transcripts and PDGFB/PDGFRB mRNA expression in dermatofibrosarcoma protuberans
}

\author{
Tomonari Takahira ${ }^{1}$, Yoshinao Oda ${ }^{1}$, Sadafumi Tamiya ${ }^{1}$, Koichi Higaki ${ }^{2}$, \\ Hidetaka Yamamoto ${ }^{1}$, Chikashi Kobayashi ${ }^{1}$, Teiyu Izumi ${ }^{1}$, Naomi Tateishi ${ }^{1}$, \\ Yukihide Iwamoto ${ }^{3}$ and Masazumi Tsuneyoshi ${ }^{1}$
}

${ }^{1}$ The Department of Anatomic Pathology, Graduate School of Medical Sciences, Kyushu University, Fukuoka, Japan; ${ }^{2}$ Department of Pathology, Saint Mary's Hospital, Kurume, Japan and ${ }^{3}$ Department of Orthopedic Surgery, Graduate School of Medical Sciences, Kyushu University, Fukuoka, Japan

\begin{abstract}
Fusion of the collagen type I alpha 1 (COL1A1) gene with the platelet-derived growth factor beta chain (PDGFB) gene has been described in dermatofibrosarcoma protuberans. The abnormal fusion transcripts probably cause PDGFB and its receptor (platelet-derived growth factor receptor beta, PDGFRB) autocrine stimulation and cell proliferation, which are responsible for the development of dermatofibrosarcoma protuberans. A reverse transcription-polymerase chain reaction assay was performed to detect the COL1A1-PDGFB fusion transcripts in 57 samples. In addition, the PDGFB gene amplification and PDGFB/PDGFRB mRNA levels were quantified by a real-time PCR system for the samples in which the fusion transcripts had been successfully detected. The fusion transcripts were detected in 42 of 57 samples. Various exons of the COL1A1 gene were fused in frame with the PDGFB gene; exons 7 and 25 were found to be slightly more frequently involved than the other exons. The PDGFB gene amplification levels varied from 0.6 to 8.3 (mean 2.4) in 42 tumor samples and from 0.4 to 3.0 (mean 1.2) in 20 adjacent normal tissue samples. In the 20 paired samples, the PDGFB gene amplification in the tumor was significantly higher than that in the normal tissue. The presence of PDGFB and PDGFRB mRNAs was demonstrated in 26 and 21 of 26 cases, respectively. The PDGFB and PDGFRB mRNA expression levels showed a good correlation $(r=0.76, P<0.0001)$. These results indicate that the fusion protein, which is processed by the COL1A1-PDGFB transcripts, can serve as a functional ligand for PDGFRB.

Modern Pathology (2007) 20, 668-675; doi:10.1038/modpathol.3800783; published online 13 April 2007
\end{abstract}

Keywords: dermatofibrosarcoma protuberans; COL1A1-PDGFB fusion transcripts; platelet-derived growth factor; platelet-derived growth factor receptor

Dermatofibrosarcoma protuberans is a dermal and subcutaneous tumor of intermediate malignancy. Tumors are slow growing and rarely metastasize, but they are prone to local recurrence after surgery. Cytogenetic features of dermatofibrosarcoma protuberans include a recurrent translocation $\mathrm{t}(17 ; 22$, q22;q13) and supernumerary ring chromosomes. Translocation 17;22 has been shown to result in a fusion of the platelet-derived growth factor beta

Correspondence: Dr Y Oda, MD, Department of Anatomic Pathology (Second Department of Pathology), Pathological Sciences, Graduate School of Medical Sciences, Kyushu University, Maidashi 3-1-1, Higashi-ku, Fukuoka 812-8582, Japan.

E-mail: oda@surgpath.med.kyushu-u.ac.jp

Received 20 December 2006; revised 6 March 2007; accepted 7 March 2007; published online 13 April 2007 chain (PDGFB) gene in 22q13 with the collagen type I alpha 1 (COL1A1) gene in 17q22. In all analyzed translocations, the breakpoints occur in the intron preceding exon 2 of the $P D G F B$ gene, whereas the COL1A1 part varies and includes various exons in the alpha-helical domain. ${ }^{1}$ The fusion deletes all known elements that negatively control $P D G F B$ transcription and translation, which are considered as oncogene-activating events. ${ }^{2}$ The abnormal fusion transcripts probably cause PDGFB and its receptor (platelet-derived growth factor receptor beta, PDGFRB) autocrine stimulation and cell proliferation, which are responsible for the development of dermatofibrosarcoma protuberans. Recently, patients with unresectable dermatofibrosarcoma protuberans have been reported to be successfully 
treated with imatinib, a potent and specific inhibitor of several protein-tyrosine kinases, including PDGFRB. ${ }^{3-6}$ Accordingly, both generations of the COL1A1-PDGFB fusion transcripts and their downstream PDGFB/PDGFRB signaling pathway are considered to play an important role in tumorigenesis and treatment of dermatofibrosarcoma protuberans. In this study, we detected the fusion gene transcripts and quantified the $P D G F B$ gene amplification and PDGFB/PDGFRB mRNA levels by using a real-time polymerase chain reaction (PCR) system.

\section{Materials and methods}

\section{Tumor Samples and Detection of Fusion Gene Transcripts}

For nucleic acid extraction, 57 consecutive cases of dermatofibrosarcoma protuberans registered between 1975 and 2004 were retrieved from the files of the Department of Anatomic Pathology, Graduate School of Medical Sciences, Kyushu University, Japan. Since written informed consent was not obtained, for strict privacy protection, identifying information for all samples was removed before analysis; this procedure was in accordance with the Ethical Guidelines for Human Genome/Gene Research enacted by the Japanese Government. Frozen samples stored at $-80^{\circ} \mathrm{C}$ were preferred because such materials would enable further genetic analyses. However, frozen materials could be obtained in only five cases. In the remaining 52 cases, the analysis was restricted to DNA and RNA extracted from the paraffin-embedded tissue. Genomic DNA was purified using standard proteinase $\mathrm{K}$ digestion and phenol/chloroform extraction. RNA was extracted from the frozen tissue according to the standard procedures. For RNA extraction from the paraffin-embedded tissue, $1 \mathrm{ml}$ xylene was added to the paraffin curls and spun in a microcentrifuge for $5 \mathrm{~min}$. Xylene was removed and the first step was repeated. The tissue was then washed twice in $1 \mathrm{ml}$ $100 \%$ cold ethanol and spun for $5 \mathrm{~min}$ at a high speed; this was followed by vacuum drying for $7 \mathrm{~min}$ and overnight digestion at $55^{\circ} \mathrm{C}$ in $0.2 \mathrm{ml} \mathrm{RNA}$ digestion buffer (20 mM Tris-HCl (pH 8.0), $20 \mathrm{mM}$ EDTA, 2\% SDS in diethyl pyrocarbonate (DEPC) water), and $10 \mu \mathrm{l}$ of $100 \mu \mathrm{g} / \mu \mathrm{l}$ proteinase K. After incubation, $1.0 \mathrm{ml}$ of Trizol reagent (Gibco BRL, Gaithersburg, MD, USA) was added. Subsequently, $200 \mu \mathrm{l}$ of chloroform was added, mixed well by vortexing, and spun at a high speed for $15 \mathrm{~min}$ at room temperature. The top aqueous RNA layer was transferred to another fresh tube and an equal volume of isopropanol was added. The content was spun for $15 \mathrm{~min}$ and the supernatant was removed, followed by washing in $70 \%$ ethanol. The dry pellet was left at room temperature for 15-20 min and 10-20 $\mu \mathrm{l}$ of DEPC water was then added. The quality of RNA was determined using an OD ratio of 1.6-1.8 and was found to be acceptable.

Reverse transcription was carried out using the SuperScript III First-Strand Synthesis System (Invitrogen, Japan), with the following modifications. Of a total volume of $20 \mu \mathrm{l}, 2 \mu \mathrm{g}$ RNA or a maximum allowable volume $(8 \mu \mathrm{l})$ of RNA when it was below the measurable level was added. The first-strand cDNA synthesis was primed using the PDGFBexon2-1 antisense primer (Table 1). To amplify the COL1A1-PDGFB fusion transcripts,

Table 1 Oligonucleotides used for RNA extraction and detection of the COL1A1-PDGFB fusion transcripts

\begin{tabular}{|c|c|c|c|}
\hline Exon & $\operatorname{Primer}\left(5^{\prime}-3^{\prime}\right)$ & Exon & $\operatorname{Primer}\left(5^{\prime}-3^{\prime}\right)$ \\
\hline \multicolumn{4}{|l|}{ COL1A1 } \\
\hline 6 & gctccccagctgtcttatgg & 31 & cggcaacgatggtgctaag \\
\hline 7 & ctctggtcctcgtggtctcc & 32 & gaacgtggtgcagctggtctt \\
\hline 8 & tcagggtgctcgaggattgc $^{a}$ & 33,34 & gccccattggtcctcctg \\
\hline 10 & aacctggtcgtcctggtgag & 35 & cagcggccctgctggt \\
\hline 12 & gtggtttggatggtgccaag & 36 & agaccgtggtgagcctggt \\
\hline 14 & cctgcctggtgagagaggtc & 37 & ctggtgctaaaggcgatgct \\
\hline 15 & ggtgctcgtggaaatgatgg $^{\mathrm{a}}$ & 38 & $\operatorname{tgctcctggagccaaaggtg}^{a}$ \\
\hline 17 & agggtgtgcgtggtgagc & 39 & ggtgctactggtttccctggt \\
\hline 19 & gctcctggcttccctggt & 40 & tgctggcgagaaaggatcccctg ${ }^{\mathrm{a}}$ \\
\hline 20 & cctggcagcaaaggagacac & 41 & gaggcttccctggtcttcct \\
\hline 21 & aggtgaacccggacccact & 42 & accccctggtgaatctggac \\
\hline 23 & aagctggtcgtcccggtgaagc $^{a}$ & 44 & tggcaagagtggtgatcgtgg \\
\hline 24 & ggcagccctggtcctgat & 46 & tggcttctctggcctccaggg $^{\mathrm{a}}$ \\
\hline 25 & caggctggtgtgatgggatt & 47 & gtccctctggagcctctggt \\
\hline 27 & gcaaagatggagaggctgga & 48 & gtctccctggccccattg \\
\hline 29 & ccaggtgaagcaggcaaacc & 49 & tgccgtgacctcaagatgtg \\
\hline \multicolumn{4}{|l|}{ PDGFB } \\
\hline Exon 2-1 & atcaaaggagcggatcgagtggtc & & \\
\hline Exon 2-2 & tggtcactcagcatctcataaagc & & \\
\hline
\end{tabular}

\footnotetext{
${ }^{\mathrm{a}}$ The same primers as previously described. ${ }^{7}$
} 
single-step PCR was carried out using a set of specific COL1A1 sense primers and the PDGFBexon2-2 antisense primer (Table 1). The COL1A1 primers corresponded to the alpha-helical domain of the COL1A1 gene (exons 6-49). Some primers were the same as described previously; ${ }^{7}$ however, other primers were designed using primer 3 (http:// frodo.wi.mit.edu/cgi-bin/primer3/primer3_www.cgi). The PCR profile consisted of 45 cycles of denaturation at $94^{\circ} \mathrm{C}$ for $1 \mathrm{~min}$, annealing at $66^{\circ} \mathrm{C}$ for $45 \mathrm{~s}$, and elongation at $72^{\circ} \mathrm{C}$ for $50 \mathrm{~s}$, followed by a final extension at $72^{\circ} \mathrm{C}$ for $10 \mathrm{~min}$. The PCR products were visualized by ethidium bromide staining on a $3 \%$ agarose gel. The DNA band was purified using a SUPREC tube (TAKARA BIO, Tokyo, Japan) and the products were reamplified for 25 cycles. The amplified product was purified by Microcon centrifugal filter devices (Millipore, Bedford, MA, USA). After purification, direct sequencing was carried out by the dideoxy chain termination method using an ABI Prism 310 sequence analyzer (Applied Biosystems, Foster City, CA, USA).

\section{Evaluation of the PDGFB Gene Amplification Level}

Real-time quantitative PCR was performed on genomic DNA extracted from 42 cases in which the COL1A1-PDGFB fusion transcripts had been successfully detected. In 20 cases, adjacent normal tissue samples were also evaluated. An SYBR green assay was used to evaluate $P D G F B$ gene amplification in comparison with a reference gene and a normal tissue. We selected the albumin gene located at 4q11-q13 as the reference gene because no genetic alteration has been detected in this chromosomal region, as determined by CGH studies. ${ }^{8-10}$ PCR was performed using the ABI Prism 7700 Sequence Detection System (Applied Biosystems) according to the manufacturer's protocol. The PDGFB gene copy numbers were calculated from the standard curve constructed from normal DNA amplification. The sequences of the primers are summarized in Table 2. The primers for amplification of the albumin gene were the same as described previously. ${ }^{11}$

\section{Evaluation of PDGFB and PDGFRB mRNA Expression}

Quantitation of mRNA was performed using the TaqMan assay. Reverse transcription procedure was carried out in almost the same manner as described above, whereas the first-strand cDNA synthesis was primed using a random hexamer. The quality of the extracted RNA was assessed by RT-PCR using primers for PGK transcripts (189bp). ${ }^{12}$ Twenty-six cases that yielded a visible PCR product on a $2 \%$ agarose gel were selected for further analysis. The primers and TaqMan probe of PDGFB, PDGFRB, and GAPDH as an endogenous control were selected from predesigned TaqMan gene expression assays (assay ID: PDGFB, Hs00234042_m1; PDGFRB, Hs00182163_m1; GAPDH, Hs99999905_m1). PCR was performed using the ABI Prism 7700 Sequence Detection System. To construct the standard curve for the quantitation of PDGFB and PDGFRB mRNA levels, we evaluated five representative osteosarcoma cell lines (MG-63, U-2/OS, SaOS-2, OST, and MNNG/HOS) because several studies have demonstrated the expression of PDGF and PDGFR in these cell lines. ${ }^{13,14}$ The standard curve was then constructed using 10-fold serially diluted total RNA extracted from the MNNG/HOS cell line, which showed the highest expression level (data not shown). The data obtained were standardized using the GAPDH data.

\section{Statistical Analysis}

A paired $t$-test was used to compare the PDGFB gene amplification level of the tumor tissue and that of the adjacent normal tissue samples. Linear regression and Pearson's correlation coefficient were used to determine correlation between the two parametric variables acquired from the quantitative PCR analyses. A $P$-value of less than 0.05 was considered statistically significant.

\section{Results}

\section{Fusion Gene Transcripts and Clinicopathological Findings}

The distribution of clinicopathological characteristics is outlined in Table 3. The COL1A1-PDGFB fusion transcripts were detected in $42(74 \%)$ of 57 samples. Five samples were obtained from recurrent tumors, whereas the remaining samples were obtained from primary tumors. Of the 42 samples, 22 were ordinary dermatofibrosarcoma protuberans and 20 were dermatofibrosarcoma protuberans-associated histological variants (10 dermatofibrosarcoma protuberans with fibrosarcomatous component

Table 2 Oligonucleotides used for evaluation of the PDGFB gene amplification

\begin{tabular}{|c|c|c|c|}
\hline & Primer $\left(5^{\prime}-3^{\prime}\right)$ & & Primer $\left(5^{\prime}-3^{\prime}\right)$ \\
\hline $\begin{array}{l}\text { PDGFB exon } 5 \mathrm{~F} \\
\text { Albumin } \mathrm{F}\end{array}$ & $\begin{array}{l}\text { acggcctgtgacccgaag } \\
\text { tgaaacatacgttcccaaagagttt }^{\text {a }}\end{array}$ & $\begin{array}{l}\text { PDGFB intron } 5 \mathrm{R} \\
\text { Albumin } \mathrm{R}\end{array}$ & $\begin{array}{l}\text { ctgagcctggaaaggtggtt } \\
\text { ctctccttctcagaaagtgtgcatat }^{\mathrm{a}}\end{array}$ \\
\hline
\end{tabular}

\footnotetext{
${ }^{\mathrm{a}}$ The same primers as previously described. ${ }^{11}$
} 
Table 3 Clinicopathological and molecular analysis data of dermatofibrosarcoma protuberans (DFSP)

\begin{tabular}{|c|c|c|c|c|c|c|c|c|c|}
\hline Case & Age & Sex & Location & $\begin{array}{l}\text { Breakpoint of } \\
\text { COL1A1 }\end{array}$ & Histology & $\begin{array}{l}\text { PDGFB gene } \\
\text { amplification } \\
\text { (tumor) }\end{array}$ & $\begin{array}{l}\text { PDGFB gene } \\
\text { amplification } \\
\text { (normal) }\end{array}$ & $\begin{array}{c}\text { mRNA level } \\
\text { of PDGFB }\end{array}$ & $\begin{array}{c}\text { mRNA level } \\
\text { of PDGFRB }\end{array}$ \\
\hline 1 & 27 & $\mathrm{~F}$ & Breast & Exon 6 & DFSP & 1.0 & 0.4 & N/A & N/A \\
\hline 2 & 48 & $\mathrm{M}$ & Clavicular region & Exon 7 & Myxoid DFSP & 1.2 & N/A & N/A & N/A \\
\hline 3 & 13 & $\mathrm{~F}$ & Groin & Exon 7 & DFSP & 1.7 & N/A & 26877 & 948 \\
\hline 4 & 31 & $\mathrm{~F}$ & Abdominal wall & Exon 7 & DFSP & 1.8 & 1.4 & 4261 & 39 \\
\hline 5 & N/A & N/A & N/A & Exon 7 & DFSP & 3.3 & N/A & N/A & N/A \\
\hline 6 & 39 & $\mathrm{M}$ & Chest wall & Exon 7 & DFSP with FS & 2.1 & 1.3 & N/A & N/A \\
\hline $7^{\mathrm{a}}$ & 81 & $\mathrm{~F}$ & Groin & Exon 13 & DFSP & 2.1 & 1.3 & 1013 & 31 \\
\hline 8 & 28 & $\mathrm{M}$ & Abdominal wall & Exon 16 & DFSP & 1.8 & 1.3 & 7 & U/L \\
\hline $9^{\mathrm{a}}$ & 66 & $\mathrm{M}$ & Groin & Exon 16 & Myxoid DFSP & 2.3 & N/A & N/A & N/A \\
\hline 10 & 36 & $\mathrm{M}$ & Chest wall & Exon 16 & DFSP & 1.4 & N/A & 114012 & 9612 \\
\hline $11^{\mathrm{a}}$ & 82 & $\mathrm{M}$ & Abdominal wall & Exon 17 & DFSP with FS & 4.3 & N/A & 275 & 3 \\
\hline 12 & 26 & M & Abdominal wall & Exon 25 & DFSP with FS & 3.1 & 1.8 & 61513 & 2413 \\
\hline 13 & 39 & $\mathrm{M}$ & Chest wall & Exon 25 & DFSP & 2.1 & 1.5 & 5583 & 336 \\
\hline 14 & 45 & $\mathrm{M}$ & Chest wall & Exon 25 & DFSP & 1.9 & 1.6 & N/A & N/A \\
\hline 15 & 61 & $\mathrm{~F}$ & Abdominal wall & Exon 25 & DFSP & 4.1 & 1.2 & 86451 & 1708 \\
\hline 16 & 47 & $\mathrm{~F}$ & Chest wall & Exon 25 & DFSP with FS & 2.3 & N/A & N/A & N/A \\
\hline 17 & 50 & $\mathrm{~F}$ & Chest wall & Exon 25 & DFSP & 1.7 & N/A & N/A & N/A \\
\hline 18 & 52 & $\mathrm{M}$ & Abdominal wall & Exon 25 & DFSP & 0.8 & N/A & 14406 & 98 \\
\hline 19 & 55 & $\mathrm{~F}$ & Abdominal wall & Exon 26 & DFSP with FS & 5.0 & N/A & N/A & N/A \\
\hline 20 & 41 & $\mathrm{M}$ & Groin & Exon 32 & DFSP & 3.5 & 1.0 & 19239 & 838 \\
\hline 21 & 31 & $\mathrm{~F}$ & Thigh & Exon 32 & DFSP & 2.8 & 1.4 & N/A & N/A \\
\hline 22 & 64 & $\mathrm{~F}$ & Abdominal wall & Exon 32 & DFSP & 2.1 & N/A & 120 & U/L \\
\hline 23 & 39 & $\mathrm{~F}$ & Back & Exon 34 & Myxoid DFSP & 3.6 & N/A & 379484 & 7605 \\
\hline 24 & 41 & M & Chest wall & Exon 35 & DFSP & 0.6 & N/A & 19007 & $\mathrm{U} / \mathrm{L}$ \\
\hline 25 & N/A & N/A & N/A & Exon 35 & DFSP & 2.0 & N/A & 30321 & 997 \\
\hline 26 & 43 & $\mathrm{~F}$ & Shoulder & Exon 37 & DFSP with FS & 2.6 & N/A & 41949 & 419 \\
\hline $27^{\mathrm{a}}$ & 28 & $\mathrm{~F}$ & Back & Exon 38 & Bednar & 5.0 & 3.0 & N/A & N/A \\
\hline 28 & 64 & $\mathrm{M}$ & Scapular region & Exon 40 & $\mathrm{SAF}^{\mathrm{c}}$ & 2.1 & N/A & 728 & U/L \\
\hline 29 & 64 & $\mathrm{~F}$ & Groin & Exon 42 & DFSP & 2.8 & 0.6 & 31834 & 3050 \\
\hline 30 & 26 & $\mathrm{~F}$ & Abdominal wall & Exon 42 & DFSP with FS & 1.8 & N/A & N/A & N/A \\
\hline 31 & 52 & $\mathrm{~F}$ & Chest wall & Exon 42 & SAF & 2.2 & N/A & N/A & N/A \\
\hline 32 & 31 & $\mathrm{~F}$ & Head & Exon 43 & Bednar & 0.6 & 1.0 & 99892 & 2308 \\
\hline 33 & 31 & $\mathrm{M}$ & Chest wall & Exon 44 & DFSP & 2.6 & 1.1 & 83134 & 2369 \\
\hline 34 & 23 & M & Groin & Exon 44 & DFSP & 1.5 & N/A & 16175 & 880 \\
\hline 35 & 51 & $\mathrm{~F}$ & Chest wall & Exon 44 & DFSP & 0.9 & 1.0 & 10063 & U/L \\
\hline 36 & 39 & $\mathrm{~F}$ & Back & Exon 46 & DFSP & 2.3 & N/A & 302 & 10 \\
\hline 37 & 35 & $\mathrm{M}$ & Back & Exon 46 & SAF & 8.3 & N/A & N/A & N/A \\
\hline 38 & 49 & $\mathrm{M}$ & Groin & Exon 47 & SAF & 2.3 & N/A & N/A & N/A \\
\hline 39 & 27 & M & Groin & Exon 47 & DFSP with FS & 0.6 & 1.1 & 28669 & 2294 \\
\hline $40^{\mathrm{a}}$ & 30 & $\mathrm{M}$ & Cheek & Exon 47 & Bednar & 2.5 & 1.1 & 703 & 109 \\
\hline 41 & 36 & $\mathrm{M}$ & Shoulder & Exon 48 & DFSP with FS & 1.0 & 0.8 & 3631 & 8 \\
\hline 42 & 26 & $\mathrm{~F}$ & Vulva & Exon 48 & DFSP & 1.7 & 0.8 & N/A & N/A \\
\hline
\end{tabular}

N/A, not available; U/L, undetectable level.

${ }^{\mathrm{a}}$ Recurrent tumors.

${ }^{\mathrm{b}}$ Dermatofibrosarcoma protuberans with fibrosarcomatous component.

${ }^{\mathrm{c}}$ Superficial adult fibrosarcoma.

(Figure 1a), four superficial adult fibrosarcoma (SAF, Figure 1b), three myxoid dermatofibrosarcoma protuberans (Figure 1c), and three Bednar tumor (Figure 1d)). Sequence analysis of the PCR products revealed that various exons of the COL1A1 gene were fused in frame with the PDGFB gene (Table 3; Figure 2). Exons 7 and 25 were found to be slightly more frequently involved than the other exons. Bednar tumor and SAF showed a tendency to have distal breakpoints (exons 38, 40, 42, 43, 46, and 47); however, the distribution was not statistically significant. Among these genetically confirmed cases, the age, sex, and tumor location of two patients were unknown. The age of the patients when the primary tumor occurred varied from 13 to 64 years, and the mean age was 40.3 years. These tumors were located on the trunk in 36 patients and on the extremities and head in two patients each.

\section{PDGFB Gene Amplification Level}

The amplification level (copy number of $P D G F B /$ copy number of albumin) varied from 0.6. to 8.3 (mean 2.4) in 42 tumor samples, and from 0.4 to 3.0 (mean 1.2) in 20 adjacent normal tissue samples (Table 3). In the 20 paired samples, the PDGFB gene amplification level in the tumor samples was 
672
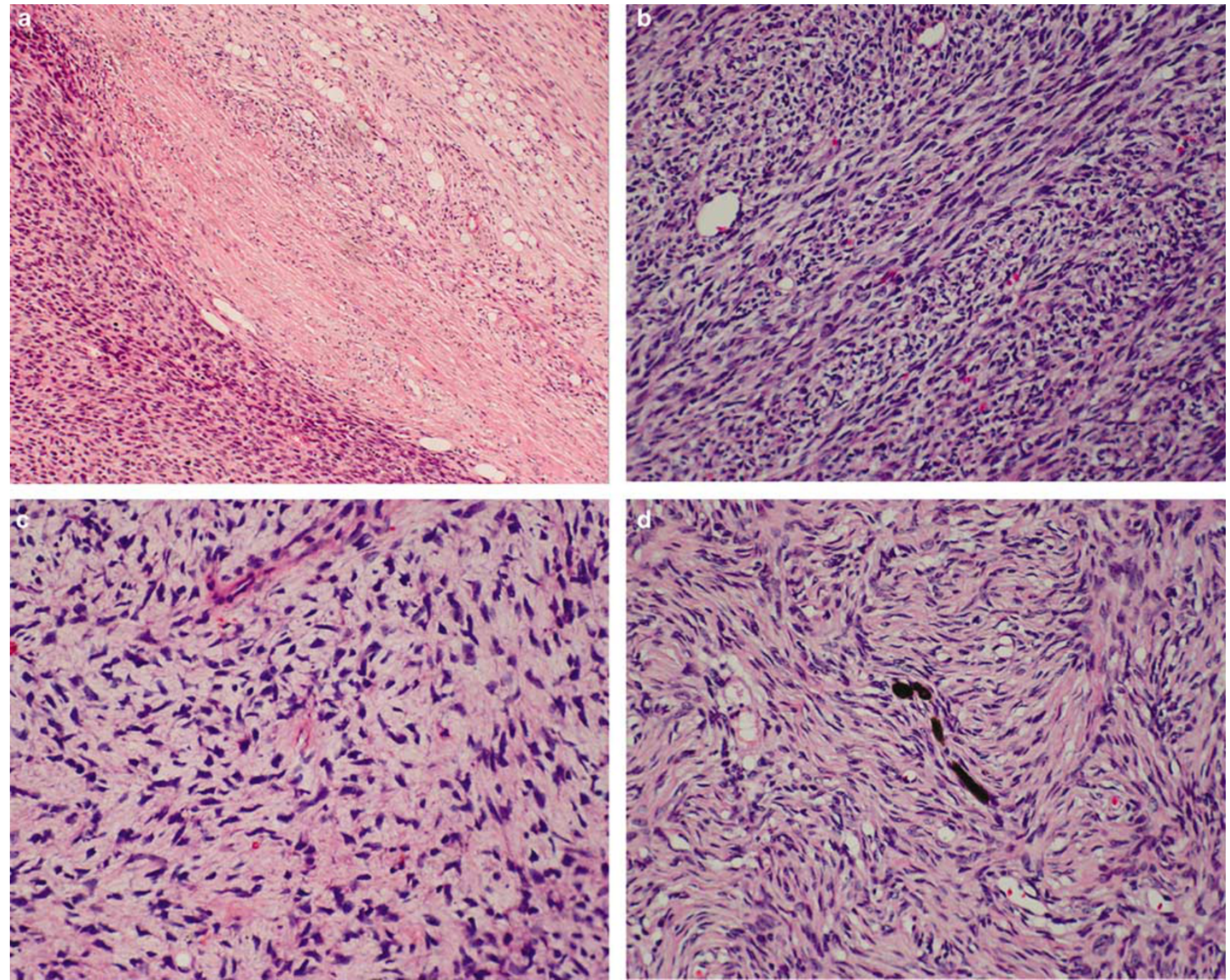

Figure 1 (a-d) Dermatofibrosarcoma protuberans-associated histological variants. (a) Dermatofibrosarcoma protuberans with a fibrosarcomatous component (case 16). This case shows an abrupt transition from ordinary dermatofibrosarcoma protuberans to the fibrosarcomatous component. (b) Superficial adult fibrosarcoma (case 38). (c) Myxoid dermatofibrosarcoma protuberans (case 23). (d) Bednar tumor (case 27).

C C TCTGGTC C T GCTGGT CCC CG A GGGG CC CC A TT C C

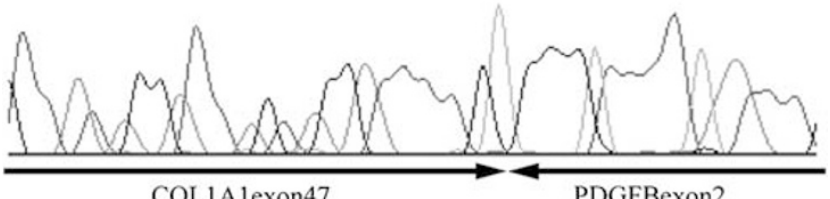

COL1Alexon47

PDGFBexon2

Figure 2 The COL1A1-PDGFB fusion transcript (case 39). The end of exon 47 in the COL1A1 gene was fused with the start of exon 2 in the PDGFB gene.

significantly higher than that in the normal tissue samples (paired $t$-test, $P=0.0002$; Figure 3). Of the 42 tumor samples analyzed, three showed a deletion (level of amplification $<0.7$ ); in two of these three samples, the amplification levels were lower than that of the paired normal tissue. A correlation was not detected between the gene amplification level and clinicopathological parameters.

\section{PDGFB and PDGFRB mRNA Expression}

The analysis of mRNA expression was performed in 26 cases with adequate RNA quality. The presence of PDGFB and PDGFRB mRNAs was demonstrated in $26(100 \%)$ and $21(81 \%)$ of 26 cases, respectively. The expression level (PDGFB or PDGFRB mRNA value/GAPDH mRNA value) varied from 120 to 379484 (mean 41525) for PDGFB expression, and from 3 to 9612 (mean 1718) for detectable PDGFRB expression. The detectable expression levels of both mRNAs showed a good correlation $(r=0.76$, $P<0.0001$; Figure 4); however, no correlation was observed between the PDGFB mRNA level and the $P D G F B$ gene amplification level $(r=0.28)$. 


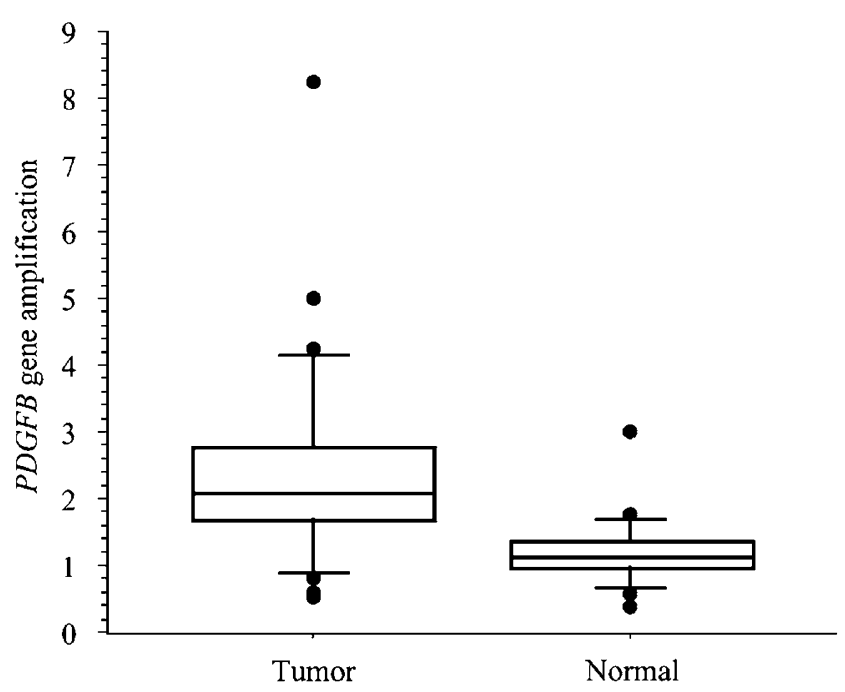

Figure 3 Box and whisker plot of the PDGFB gene amplification of the tumor samples and the adjacent normal tissue samples $(n=20)$. A statistically significant difference was observed between the two groups (paired $t$-test, $P=0.0002$ ).

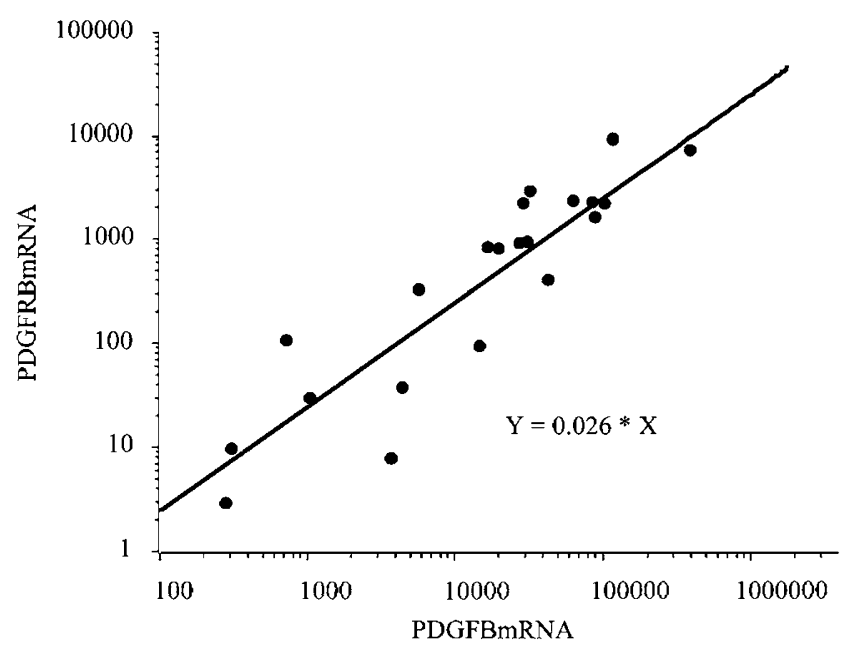

Figure 4 Relationship between the PDGFB and PDGFRB mRNA expression levels ( $n=21 ; r=0.76, P<0.0001$, logarithmic scale).

\section{Discussion}

Karyotypic analyses of dermatofibrosarcoma protuberans have shown recurrent aberrations of chromosomes 17 and 22 and have led to the molecular definition of a characteristic rearrangement that fuses various exons of the COL1A1 gene to exon 2 of the PDGFB gene. The COL1A1-PDGFB fusion transcripts have also been detected in the cells of tumors closely related to dermatofibrosarcoma protuberans, giant cell fibroblastoma, ${ }^{2,15}$ Bednar tumor, ${ }^{7}$ granular cell variant of dermatofibrosarcoma protuberans, ${ }^{16}$ and SAF. ${ }^{17}$ It has been reported that in cases with a fibrosarcomatous area in ordinary dermatofibrosarcoma protuberans or with fibrosarcoma in the recurrent or metastatic lesion, the tumor shows an aggressive clinical course. ${ }^{18-23}$ By using a microdissection technique, Wang et $a l^{24}$ detected identical COL1A1-PDGFB fusion transcripts in the two cellular components of dermatofibrosarcoma protuberans with fibrosarcomatous areas. In the present study, we detected COL1A1-PDGFB fusion transcripts in 42 of 57 cases that included 10 dermatofibrosarcoma protuberans with fibrosarcomatous areas, four SAF, three myxoid dermatofibrosarcoma protuberans, and three Bednar tumor. To our knowledge, this is the largest series of cases in which the COL1A1-PDGFB fusion transcripts were detected. Various exons in the alpha-helical domain of the COL1A1 gene have been shown to be involved in the fusion with exon 2 of the $P D G F B$ gene; in the present study, exons 7 and 25 were found to be slightly more frequently involved than the other exons. Bednar tumor and SAF showed a tendency to have distal breakpoints; however, the number of cases with these histologic subtypes was too small.

The COL1A1 gene encodes the major component of type I collagen, which is produced primarily by fibroblasts. The PDGFB protein is a potent mitogen for cells expressing functional PDGF receptors, typically cells of mesenchymal origin. The COL1A1-PDGFB fusion transcript is translated to form a precursor fusion protein that is processed into a mature and fully functional PDGFB proteina ligand for PDGFRB. ${ }^{25,26}$ Consequently, the abnormal fusion transcripts probably cause autocrine stimulation and cell proliferation, which are responsible for the development of dermatofibrosarcoma protuberans. To evaluate the importance of the autocrine signaling of PDGFB in dermatofibrosarcoma protuberans, in the present study, we quantified the PDGFB copy numbers and mRNA expression by using a real-time PCR system. We showed that the $P D G F B$ gene amplification level in the tumor samples was significantly higher than that in the normal tissue samples. Our results were confirmed by similar results obtained in some CGH-based studies, which showed the $t(17 ; 22)$ translocation is usually associated with increased copy numbers of the COL1A1 and PDGFB genes. ${ }^{8-10,27}$ Interestingly, of the 42 tumor samples analyzed, three showed a deletion; in two of these three samples, the amplification levels were lower than that of the paired normal tissue. These cases were suggested to have unbalanced translocations instead of the supernumerary ring chromosome. Although all available samples showed PDGFB mRNA expression, the expression level widely varied and was not related to the COL1A1 gene breakpoint. Gene amplification often results in an increased expression from the amplified locus (gene dosage effect). However, the present study showed no correlation between the gene amplification and mRNA expression levels of PDGFB. Two possible reasons were suggested to explain this discrepancy. The gene amplification level of the PDGFB gene in dermatofibrosarcoma protuberans is relatively low when 
compared with the amplification observed in other tumors such as amplification of the MYCN gene in neuroblastoma or that of the ERBB2 gene in breast carcinoma; these tumors frequently contain 20-50 copies of the amplified gene per cell. Consequently, the gene dosage effect in dermatofibrosarcoma protuberans was considered to be relatively limited. Another reason is the existence of other mechanisms that regulate PDGFB mRNA expression in addition to gene amplification. As mentioned above, the $P D G F B$ segment of the fusion transcript starts with exon 2, placing the $P D G F B$ gene under the control of the COL1A1 promoter, and removing all known elements that negatively control $P D G F B$ transcription and translation. It is known that the COL1A1 promoter activity is sensitive to DNA methylation and the COL1A1 gene is methylated in some human cancer cells. ${ }^{28}$ Such epigenetic change could affect the transcription of the COL1A1-PDGFB fusion gene.

The mRNA expression levels of PDGFB and PDGFRB showed a good correlation. This indicates that the fusion protein, which is processed by the COL1A1-PDGFB transcripts, functions as a ligand for PDGFRB. The expression level varied, and in our PCR system, the expression level was below the detectable level in five of 26 available cases. There was no correlation between the PDGFRB expression level and histologic subtype of dermatofibrosarcoma protuberans. As mentioned earlier, dermatofibrosarcoma protuberans with fibrosarcomatous areas shows more aggressive clinical course than ordinary dermatofibrosarcoma protuberans. Histologically, dermatofibrosarcoma protuberans with fibrosarcomatous areas shows higher proliferative activity (higher mitotic rate or MIB-1 labeling index) than ordinary dermatofibrosarcoma protuberans. ${ }^{23,29,30}$ Since no correlation was observed between the mRNA expression level of PDGFB/PDGFRB and the histologic subtype, the fibrosarcomatous transformation of dermatofibrosarcoma protuberans is thought to be caused by mechanisms such as the TP53 gene mutation ${ }^{29,31}$ or microsatellite instability $^{31}$ rather than the generation of the fusion gene and the activation of its downstream PDGFRB signaling pathway.

The optimal treatment option for dermatofibrosarcoma protuberans is complete surgical resection with wide margins. Recently, a limited number of clinical reports have suggested that imatinib, a potent and specific inhibitor of several protein-tyrosine kinases, including PDGFRB, can induce regression in patients with unresectable, recurrent, or metastatic dermatofibrosarcoma protuberans. ${ }^{3-6}$ Maki et $a l^{3}$ and McArthur et $a l^{5}$ demonstrated that cases with no evidence of $\mathrm{t}(17 ; 22)$ showed poor response to imatinib. However, some cases that carried a definite $t(17 ; 22)$ also showed a relatively poor response. Our study did not include patients treated with imatinib; therefore, further studies are needed to clarify the relationship between the clinical response to imatinib and PDGFRB expression level.
In summary, we detected the COL1A1-PDGFB fusion transcripts by using tumor specimens from 42 patients with dermatofibrosarcoma protuberans and dermatofibrosarcoma protuberans-related tumors. In addition, we investigated gene copy numbers of the $P D G F B$ gene and the expression level of PDGFB/PDGFRB mRNA by using a quantitative real-time PCR system. The gene copy numbers of the tumor samples were significantly increased than those of the adjacent normal tissue samples. PDGFB mRNA expression was detected in all analyzed samples and a correlation was observed between the PDGFB and PDGFRB mRNA expression. These results indicate that the fusion protein, which is processed by the COL1A1-PDGFB transcripts, functions as a ligand for PDGFRB. This study demonstrated that the generation of the COL1A1-PDGFB fusion transcripts and the activation of the downstream PDGFB/PDGFRB signaling pathway were involved in tumorigenesis of dermatofibrosarcoma protuberans.

\section{Acknowledgement}

This work was supported in part by a Grant-in-Aid for Scientific Research (C) (18590332) from the Japan Society for the Promotion of Science.

\section{References}

1 Sirvent N, Maire G, Pedeutour F. Genetics of dermatofibrosarcoma protuberans family of tumors: from ring chromosomes to tyrosine kinase inhibitor treatment. Genes Chromosomes Cancer 2003;37:1-19.

2 Simon MP, Pedeutour F, Sirvent N, et al. Deregulation of the platelet-derived growth factor B-chain gene via fusion with collagen gene COL1A1 in dermatofibrosarcoma protuberans and giant-cell fibroblastoma. Nat Genet 1997;15:95-98.

3 Maki RG, Awan RA, Dixon RH, et al. Differential sensitivity to imatinib of 2 patients with metastatic sarcoma arising from dermatofibrosarcoma protuberans. Int J Cancer 2002;100:623-626.

4 Price VE, Fletcher JA, Zielenska M, et al. Imatinib mesylate: an attractive alternative in young children with large, surgically challenging dermatofibrosarcoma protuberans. Pediatr Blood Cancer 2005;44:511-515.

5 McArthur GA, Demetri GD, van Oosterom A, et al. Molecular and clinical analysis of locally advanced dermatofibrosarcoma protuberans treated with imatinib: Imatinib Target Exploration Consortium Study B2225. J Clin Oncol 2005;23:866-873.

6 Labropoulos SV, Fletcher JA, Oliveira AM, et al. Sustained complete remission of metastatic dermatofibrosarcoma protuberans with imatinib mesylate. Anticancer Drugs 2005;16:461-466.

7 Wang J, Hisaoka M, Shimajiri S, et al. Detection of COL1A1-PDGFB fusion transcripts in dermatofibrosarcoma protuberans by reverse transcription-polymerase chain reaction using archival formalin-fixed, paraffinembedded tissues. Diagn Mol Pathol 1999;8:113-119. 
8 Kiuru-Kuhlefelt S, El-Rifai W, Fanburg-Smith J, et al. Concomitant DNA copy number amplification at $17 \mathrm{q}$ and $22 \mathrm{q}$ in dermatofibrosarcoma protuberans. Cytogenet Cell Genet 2001;92:192-195.

9 Nishio J, Iwasaki H, Ohjimi Y, et al. Overrepresentation of 17q22-qter and $22 q 13$ in dermatofibrosarcoma protuberans but not in dermatofibroma: a comparative genomic hybridization study. Cancer Genet Cytogenet 2002;132:102-108.

10 Linn SC, West RB, Pollack JR, et al. Gene expression patterns and gene copy number changes in dermatofibrosarcoma protuberans. Am J Pathol 2003;163: 2383-2395.

11 Hostein I, Pelmus M, Aurias A, et al. Evaluation of MDM2 and CDK4 amplification by real-time PCR on paraffin wax-embedded material: a potential tool for the diagnosis of atypical lipomatous tumours/ well-differentiated liposarcomas. J Pathol 2004;202: 95-102.

12 Jin L, Majerus J, Oliveira A, et al. Detection of fusion gene transcripts in fresh-frozen and formalin-fixed paraffin-embedded tissue sections of soft-tissue sarcomas after laser capture microdissection and rt-PCR. Diagn Mol Pathol 2003;12:224-230.

13 Benini S, Baldini N, Manara MC, et al. Redundancy of autocrine loops in human osteosarcoma cells. Int J Cancer 1999;80:581-588.

14 McGary EC, Weber K, Mills L, et al. Inhibition of platelet-derived growth factor-mediated proliferation of osteosarcoma cells by the novel tyrosine kinase inhibitor STI571. Clin Cancer Res 2002;8:3584-3591.

15 O’Brien KP, Seroussi E, Dal Cin P, et al. Various regions within the alpha-helical domain of the COL1A1 gene are fused to the second exon of the PDGFB gene in dermatofibrosarcomas and giant-cell fibroblastomas. Genes Chromosomes Cancer 1998;23:187-193.

16 Maire G, Pedeutour F, Coindre JM. COL1A1-PDGFB gene fusion demonstrates a common histogenetic origin for dermatofibrosarcoma protuberans and its granular cell variant. Am J Surg Pathol 2002;26: 932-937.

17 Sheng WQ, Hashimoto H, Okamoto S, et al. Expression of COL1A1-PDGFB fusion transcripts in superficial adult fibrosarcoma suggests a close relationship to dermatofibrosarcoma protuberans. J Pathol 2001;194: 88-94.

18 Connelly JH, Evans HL. Dermatofibrosarcoma protuberans. A clinicopathologic review with emphasis on fibrosarcomatous areas. Am J Surg Pathol 1992;16: 921-925.

19 Ding J, Hashimoto H, Enjoji M. Dermatofibrosarcoma protuberans with fibrosarcomatous areas. A clinico- pathologic study of nine cases and a comparison with allied tumors. Cancer 1989;64:721-729.

20 Mentzel T, Beham A, Katenkamp D, et al. Fibrosarcomatous ('high-grade') dermatofibrosarcoma protuberans: clinicopathologic and immunohistochemical study of a series of 41 cases with emphasis on prognostic significance. Am J Surg Pathol 1998;22:576-587.

21 O'Connell JX, Trotter MJ. Fibrosarcomatous dermatofibrosarcoma protuberans with myofibroblastic differentiaion: a histologically distinctive variant (corrected). Mod Pathol 1996;9:273-278.

22 Wrotnowski U, Cooper PH, Shmookler BM. Fibrosarcomatous change in dermatofibrosarcoma protuberans. Am J Surg Pathol 1988;12:287-293.

23 Abbott JJ, Oliveira AM, Nascimento AG. The prognostic significance of fibrosarcomatous transformation in dermatofibrosarcoma protuberans. Am J Surg Pathol 2006;30:436-443.

24 Wang J, Morimitsu Y, Okamoto S, et al. COL1A1PDGFB fusion transcripts in fibrosarcomatous areas of six dermatofibrosarcomas protuberans. J Mol Diagn 2000;2:47-52.

25 Shimizu A, O’Brien KP, Sjoblom T, et al. The dermatofibrosarcoma protuberans-associated collagen type I alpha 1/platelet-derived growth factor (PDGF) Bchain fusion gene generates a transforming protein that is processed to functional PDGF-BB. Cancer Res 1999;59:3719-3723.

26 Simon MP, Navarro M, Roux D, et al. Structural and functional analysis of a chimeric protein COL1A1PDGFB generated by the translocation $t(17 ; 22)$ (q22;q13.1) in dermatofibrosarcoma protuberans (DP). Oncogene 2001;20:2965-2975.

27 Naeem R, Lux ML, Huang SF, et al. Ring chromosomes in dermatofibrosarcoma protuberans are composed of interspersed sequences from chromosomes 17 and 22 . Am J Pathol 1995;147:1553-1558.

28 Sengupta P, Xu Y, Wang L, et al. Collagen alpha1(I) gene (COL1A1) is repressed by RFX family. J Biol Chem 2005;280:21004-21014.

29 Hisaoka M, Okamoto S, Morimitsu Y, et al. Dermatofibrosarcoma protuberans with fibrosarcomatous areas. Molecular abnormalities of the p53 pathway in fibrosarcomatous transformation of dermatofibrosarcoma protuberans. Virchows Arch 1998;433:323-329.

30 Szollosi Z, Nemes Z. Transformed dermatofibrosarcoma protuberans: a clinicopathological study of eight cases. J Clin Pathol 2005;58:751-756.

31 Takahira T, Oda Y, Tamiya S, et al. Microsatellite instability and p53 mutation associated with tumor progression in dermatofibrosarcoma protuberans. Hum Pathol 2004;35:240-245. 